\section{Phenomenal Properties of the Domain Ensembles in Proteins}

\section{Abstract}

The method of gravitational mass spectroscopy was applied to study the longrange order in collagens and gelatins at the level of domains up to 210 million Daltons. Ensembles of domains were found in both kinds of substances. Domains as ensembles of atomic nuclei masses were formed and strongly determined under the influence of gravitational noises emerged from the sun system and our galaxy. The domain distribution in ensembles in native collagens was observed to be very different from that one in the gelatins. The interaction of the polymer domains with water clusters was investigated. The dynamics of the domain ensembles in swelling, drying, crystallization and interaction with specific water morphism was understood. For some domains, a hypothetical structure was proposed. Resonance fields caused by domain ensembles in proteins and their interaction with analogues at long distances were described.

Keywords: Protein; Domain ensembles; Properties; Structure; Remote communication

Abbreviations: $\mathrm{D}_{c}$ : Part of Collapsed Structures in the Selected Mass Ensemble \%.; $f$ : Part of a Single Domain in the Selected Mass Ensemble; GMS: Gravitational Mass Spectroscopy; GN: Gravitational Noises; LRO: Long-Range Order; $\mathrm{M}_{\mathrm{GMS}}$ : Average Molecular Mass of the Whole Domain in the Selected Mass Ensemble Da; N: Total Number of Signals from Domains and Clusters in the Selected Mass Ensemble; NC1: Globular Domain in Collagen; RASC: Gly 22\%, Pro \% 13\%, Hyp 10 \%, Glu 9.8 $\%$, Ala $8 \%$, Arg $7.6 \%$ (weight \%); $W_{\mathrm{x}}$ : Added Fractions of Domain Parts \%

\section{Kristina Zubow ${ }^{1}$, Viktor Anatolievich Zubow ${ }^{1}$ and Anatolij Zubow ${ }^{2}$}

\author{
1 Research and Development, Zubow \\ Consulting, Germany \\ 2 Department of Telecommunication \\ Networks Group, TU Berlin, Germany
}

Corresponding author:

Dr. N. Kristina Zubow

” aist@zubow.de

Research and Development, Zubows Consulting, Peenehagen, Meclkenburg Vorpommern, Germany.

Tel: 493031423819

Citation: Zubow K, Zubow VA, Zubow A. Phenomenal Properties of the Domain Ensembles in Proteins. Biochem Mol Biol J. 2016, 2:1.

Received: February 10, 2016; Accepted: April 06, 2016; Published: April 11, 2016

\section{Introduction}

Previously, we have shown that molecules as ensembles of atomic nuclei, secondary and higher structures in polymers (domain concentrations) and clusters in liquids (long-range order, LRO) are formed under the influence of local gravitational noises (GN) that they are resulted from gravitational fluctuations in the sun system and in our galaxy [1]. Attempts to investigate the domain structure of gelatins and collagens did not get any satisfactory results [2]. The methods were very limited [3-6] and actively influenced the real LRO in gels [7-10]. Therefore, to acquire a true idea about the domain structure and its distribution in the amorphous polymer gels was unsuccessfully. This is especially regarded to the chains' endings of the triple helices (Figure 1) the role of these heads in physical and chemical processes such as hydrolysis, aging, oxidation. Regarding the native structure of the heads there is no clear idea [11] in the literature, but there is an understanding that they are represented by an amino group on the one side and a carboxyl one on the other side. These groups are able to form domain structures with distinct properties, typical for domains [1].

The interaction of water with collagen at the level of the configuration features of the polymer chains was studied in many works $[12,13]$ however, at this level when secondary and higher structures of protein chains interact with water cluster ensembles no reliable information can be obtained.

Since water and collagen are characterized by different cohesive energies they primarily interact among themselves cohesively leading to ensembles of clusters and domains and only then sorption processes take place. Moreover there is no information on how water with a specific LRO (water polymorphism) influences gelation processes and aging of gels, although the strong influence of some morphism water states on the energy dissolution is known [1]. 


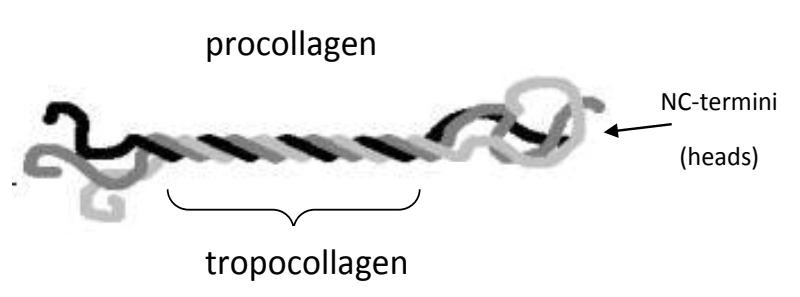

Figure 1 The structure of procollagen.

Till the authors of the present work developed the GMS as a passive analysis method for the investigation of cluster and domain structures in amorphous molecular matter $[1,14]$ there were only active methods that destroyed the native structure. The said method allows registering $\mathrm{GN}$ from atomic nucleus concentrations without affecting the external energy.

Collagens are natural polymers with a heterogeneous structure they consist of atomic nucleus concentrations (domains) of different nature. On the other site, atomic nucleus concentrations (clusters) in water have to be understood as different polymorphism states of the liquid (LRO). When water is heated up, LRO shall be destroyed and the released individual molecules can interact with the polymer fragments [15]. Water cluster ensembles in biometrics with native collagen play an important role in stabilizing the entire ensemble of nucleus masses as an unit, regardless of their nature [1], however, under an enforced impact of single water molecules on the polymer fibrils denaturation and even linking of chains [16] with the emergence of a new state of domains in gelatin could take place. Moreover to De Simone water stabilizes secondary structures of proteins [17].

Earlier we found the remote interaction of individual domains and clusters in water and synthetic polymers at large distances $[1,18]$, it was therefore, of interest to look for this effect in the domain ensembles of the simplest proteins e.g. gelatin.

The aim of this work was to study the long-range order in collagens and gelatins, the dynamics of secondary and higher structures of polymer chains in gelatins during their interaction with water cluster ensembles further, to investigate the interaction between distant domain ensembles in proteins.

\section{Materials and Methods}

Fresh native collagen from air bubble of a young fish (1 month) and from fresh sinew (approx. $86 \%$ collagen) of two year old ducks as well as industrial gelatin (Dr Oetker, FRG) were measured by the GMS method described in [1] using weak shock waves and the Zubow constant $Z=6.45 \mathrm{E}-15 \mathrm{~N} / \mathrm{m}$. The GMS method registers molecular masses (clusters and domains) direct (in vitro or in vivo) during some secunds. In Figure 2, a schematic experimental description for the study of the interaction of distant domains in protein ensembles was given.

\section{Result and Discussion}

In the GMS spectrum of the fresh fish air bubble biometric, containing native collagen, all mass concentrations of water,

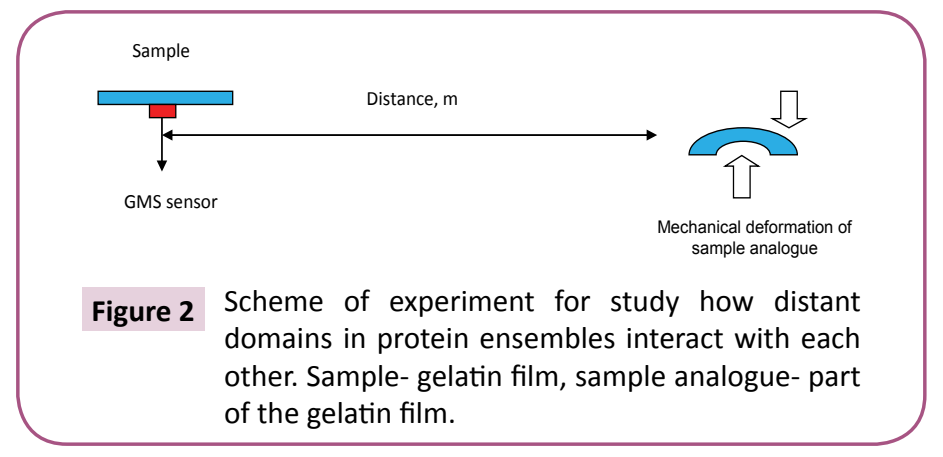

biopolymers, salts, fat and other components were found; they were represented by one single harmonious ensemble (Figure 2). The first four signals in the left part of the spectrum at 199, 1821, 5073 and 9894 Daltons were related to the main components water clusters [1] and small domains in the polymer [19] (Table 1).

The globular domains NC1 were described in numerous papers [20-24], and their masses vary over a wide range. In Figure 3, the signals of these domains [monomers (approx. $20 \ldots 35 \mathrm{kDa}$ ), dimers, trimers, hexamers ( $170 \mathrm{kDa}$ ), etc.] were marked in red, and the full range by a bracket. The signals from very large domains (>200 kDa) reflected configurations and conformational irregularities in polymer chains (loops, spirals, coils) moreover, oscillations of secondary and higher structures as well as of micelles and sub-micelles [25]. Additionally, the spectrum showed clear distinctions between the expanded $(+f)$ and collapsed $(-f)$ forms of domains at 1,905 kDa (broad arrow) and 122,253 $\mathrm{kDa}$ (dotted arrow). The very large sub ensemble of oscillators, with a maximum at $158,458 \mathrm{kDa}$ was assigned to super micelle structures. Thus, the structure of this native collagen was represented by a highly organized ensemble of domains.

The distribution of clusters and domains in fresh biomatrix of sinew from a two-year-old duck was given in Figure 4.

As shown in Figures $\mathbf{3}$ and $\mathbf{4}$ the domain structures in air bubble and sinew strongly differed from each other regarding both the number of domains and their distribution in the ensemble as well as their physical characteristics (e.g. part of domains in collapsed form). It is believed that the differences are due to aging processes in collagen, degradation of domains that were weakly integrated in the oscillation with neighbors and the maintenance of the main structure-forming domains or their sub ensembles. In the area of head polymer oscillation, the strong signals from NC1 disappeared, but there was a "poor" family of expanded hexamers (170.2 kDa, wide vertical arrow), directly evidencing the destruction of collagen. The signal at $24.5 \mathrm{kDa}$ could reflect the presence of NC1 monomers (arrow), and the strong one at $815 \mathrm{Da}$ (indicated by an asterisk) the domains of 14 glycine units in a spiral shape or the domains of five repeating average structures in collagen chains (RASC) consisting of: Gly 22 -Pro \% 13\% - Hyp $10 \%$ - Glu $9.8 \%$ - Ala $8 \%$ - Arg $7.6 \%$ (weight \%).

Industrial gelatins were obtained by extraction of collagen from different animal row materials. In Figure 5, the spectrum of a food gelatin was given, nearly all domains were represented in their expanded form $(+f)$. The strong signal at $455 \mathrm{Da}$ should be assigned to small spirals of 2 meanders each of which consists of 
Table 1 Models of the known water clusters and small domains in collagen.

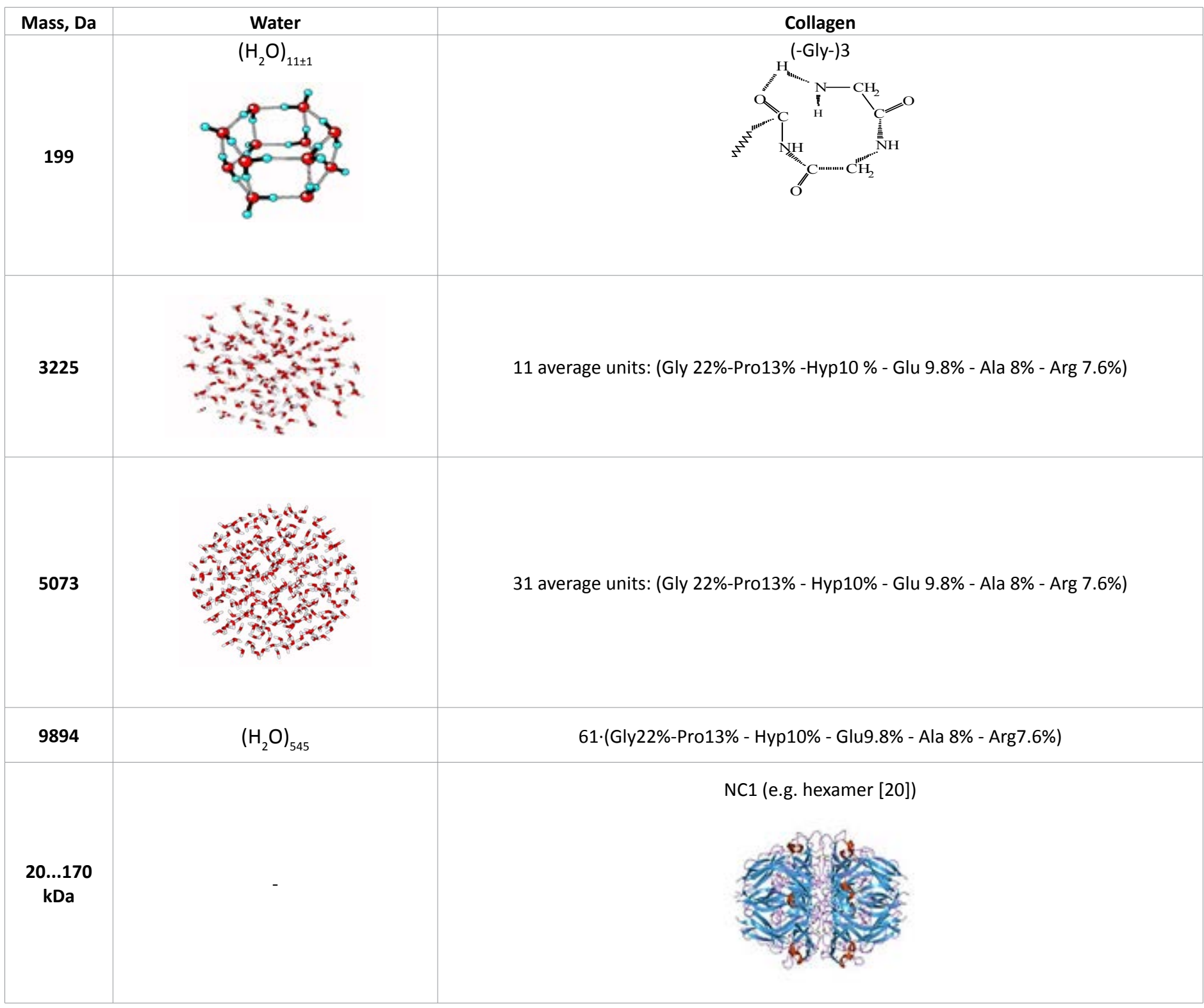

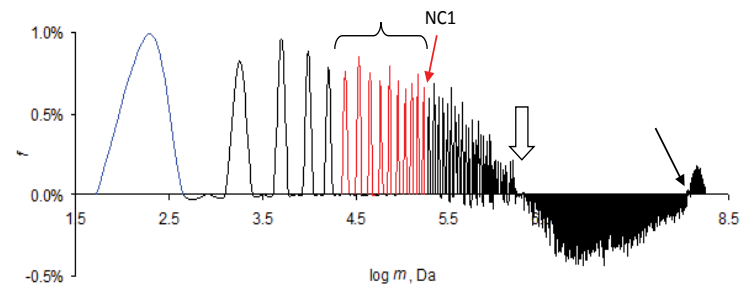

Figure 33 GMS spectrum of a fresh fish air bubble from a young roaches (1 month). Average molecular mass of the whole ensemble $M_{G M S}=33,372 \mathrm{kDa}$, total number of signals from domains and clusters $\mathrm{N}=496$, part of collapsed structures $\mathrm{D}_{\mathrm{c}}=68 \%$. Weak shock waves [1]. The base water cluster is given in the left part of the spectrum NC1=170,251 Da. $\mathrm{Z}=6.45 \mathrm{E}-15 \mathrm{~N} / \mathrm{m}, \mathrm{m}$ : mass in Dalton; $f$ : part of a single domain in ensemble. Photo of fibrils of native collagen taken from [25].

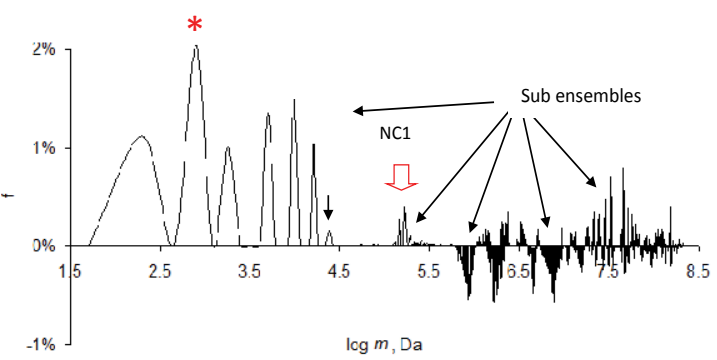

Figure 4 GMS spectrum of fresh sinew from a two-yearold domestic duck. $M_{G M S}=40,275 \mathrm{kDa}, \mathrm{N}=1643$, $D_{c}=53 \%$. Weak shock waves. Above some tertiary protein structures were given for understanding.

4 Gly units or 3 meanders of RASC in expanded form. This signal was found in dry gelatin films, too. It could belong to the water cluster $\left(\mathrm{H}_{2} \mathrm{O}\right)_{25}$ however, it is known in theory, only, throughout 


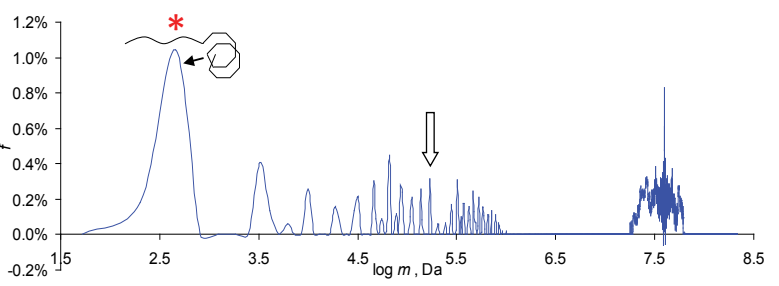

Figure $5 \mathrm{GMS}$ spectrum of industrial food gelatin (film). $\mathrm{M}_{\mathrm{GMS}}=34,585 \mathrm{kDa}, \mathrm{N}=566, \mathrm{D}_{\mathrm{c}}=0 \%$. The signal $\mathrm{NC1}$ at 170.251 Da was marked with a broad arrow, the domain of $455 \mathrm{Da}$ - with asterisk.

our research, it has never been detected [1]. The next signal at $\log \mathrm{m}=3.5$ was found in solid gelatins and gelatin hydrogels, but not in native collagens it corresponds to 179 molecules of water or 20 RASC. A sub ensemble of huge domains with a peak at $39,382 \mathrm{kDa}$ ( $\log \mathrm{m}=7.5$ ) was typical for the series of studied food gelatins, to heat, water and aging, it was more or less resistant. It has to be understood as oscillations of giant globular super micelle structures. The signals in the area of smaller domains were variable, here the spectrum depended on the origin of the gel.

For understanding the state of domain ensembles in proteins the integrated curves of domain distribution were analysed (Figure 6). Here the industrial food proteins strongly differed from native collagens where the distribution was more uniformly. Attempts to convert higher polymer chain structures in gelatins to native collagens were carried out applying various procedures of water removing. As reported by Belitz [11] the restructuring of polymer chains in gelatins to collagen is possible in concentrated gels at low temperatures. On the other hand, seed crystals are used in the crystal chemistry to achieve regular structures. Probably, the domain ensembles in gelatins may act as seed centres for developing native collagen structures or at least their precursors in processes of drying or saturation with water. It is difficult to conceive that industrial gelatin shall be completely converted to native collagen, but a maximum approach to a long-range order could be tried at least.

In Figure $\mathbf{7}$ it was shown how changed the distribution of domains in gelatin during drying. This process was accompanied by not only the removal of water from the gelatin matrix, but also intense restructuring processes within the whole ensemble of protein domains. Here, the strongest signals were assigned to the small domains of log $m<3.5$ where the domain forms were in strong dependence on the reconstruction processes in secondary and higher structures of the polymer chains of $\log m>4.5$. The signal at $\log m=2.3$ indicated either on the water basic cluster of $11 \pm 1$ molecules or on the domain in the chain ends of type like below:

After drying the film this signal remained therefore, it shout be attributed to this domain in collagen (Figure 3 ). The next strong signal at $\log m=3.1$ appeared very seldom, it was ascribed to the domain in the expanded form with a mass of $1257 \mathrm{Da}$ consisting of 8 RASC. This oscillator can be believed of as a spiral of 2 meanders, each of which contains 4 RASC units. It has to be mentioned that in duck collagen (Figure 3) strong signals

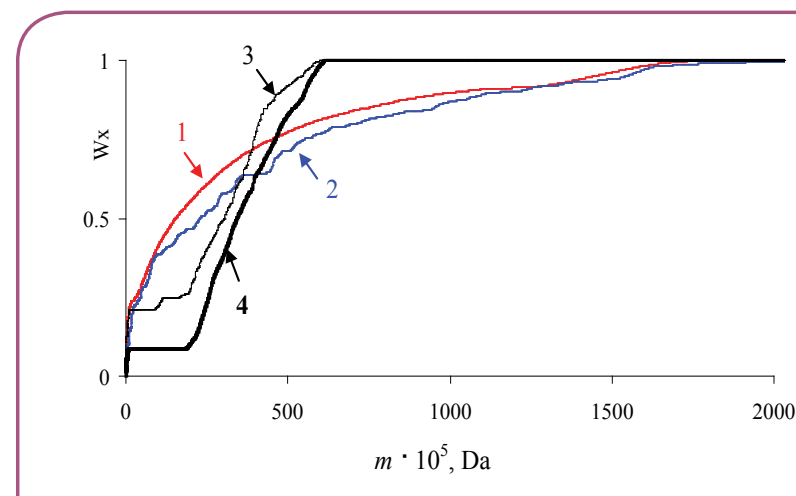

Figure 6 Integral curves of added fractions of domain parts $\left(W_{\mathrm{x}}\right)$ in proteins. 1: native collagen of air bubble (Figure 2), 2: native collagen of sinew (2-year-old duck, Figure 3 ), 3: food gelatin (Figure 5) and 4: another charge of (3).

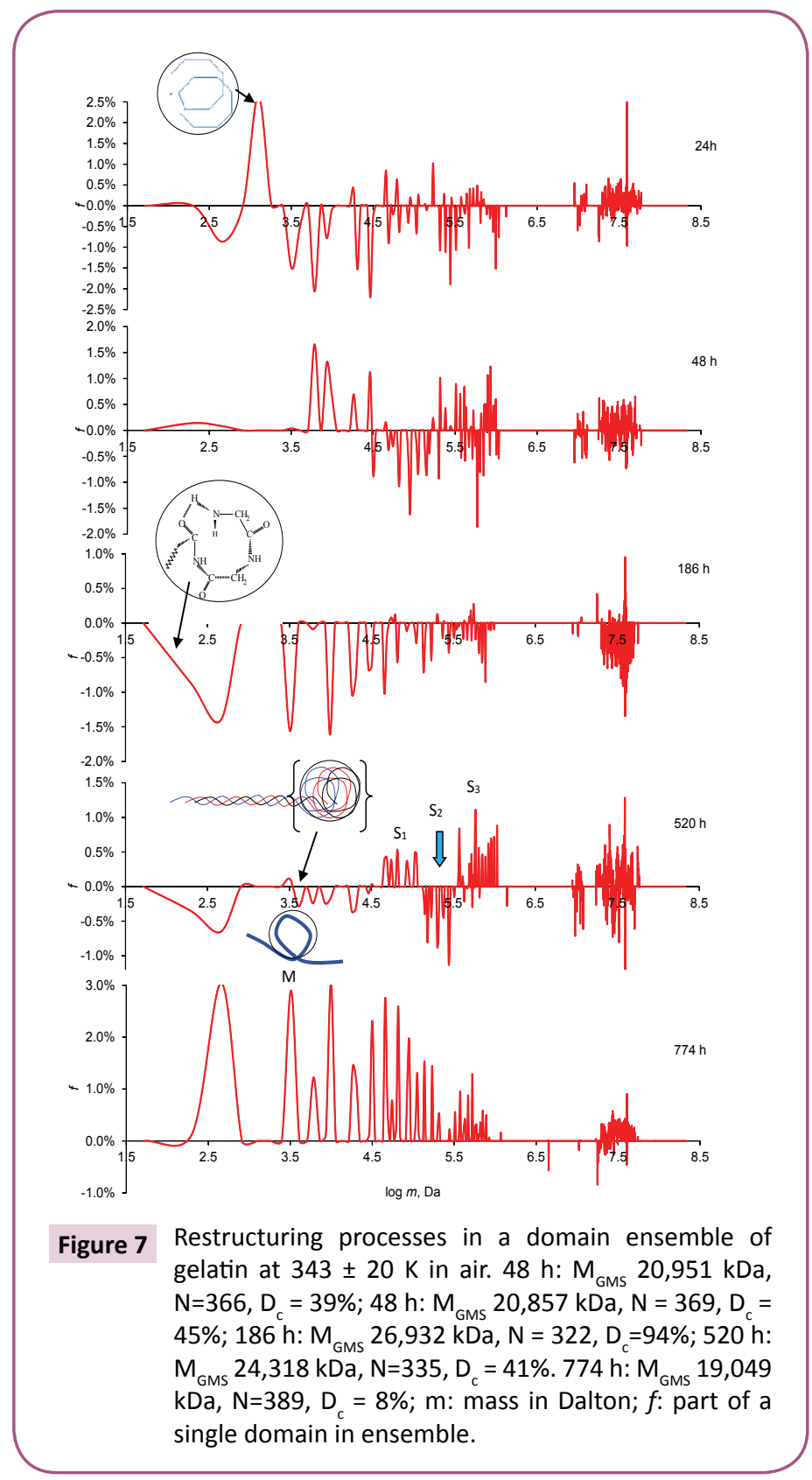


of domains with similar masses ( $\log m=2.91$ and $\log m=3.26$ ) were detected, but not this signal. In air bubble collagen, however, (Figure 3) all these signals disappeared. Therefore, it was believed that all these signals in gelatin reflected domains belonging to the end groups in the polymer chains. The signal at $\log m=3.5$ changing sporadically its characteristics, was assumed to play a key role in the reorganization of the whole ensemble. The signal from domain (M) of 20 RASC with 3,225 Da could be emerged by an oscillating loop with a diameter of nearly 1.5 $\mathrm{nm}$, tensions of which $(f)$ reflect the state of higher structures in polymer chains. In the area of very large domains (log $m>6.5$ ), evident changes in the sub ensemble at gel dehydration were observed at the intermediate stages of drying only. These were likely network collagens ( $\log \mathrm{m}^{\sim 7.5}$ ) of IV, VIII, X types that were difficult to transfer to another state, to destroy or to crystallize. For this sample of gelatin, the stability was maintained both at long heating, swelling and on exposure to specific polymorphic forms of water (acoustic LRO stimulations), (Figures 4,6,11,13).

In the protein film, after $520 \mathrm{~h}$ sub ensembles were formed, two from expanded domains $(+f)$ in S1 and S3 and one from collapsed $(-f)$ ones in $\mathrm{S}_{2}$. They reflected the beginning of the crystallization of chains and their fragments with similar cohesive energies. The appearance of these sub ensembles with a strong individuality may indicate on the number of different sources of raw material for the food gelatin production and consequently, on the number of procollagen kinds (super structures with different cohesion energies). The signals from small domains were sharply weakened, showing that these small domains were involved in the structures of sub ensembles. Here was interesting to note that the domain $4097 \mathrm{kDa}$ appeared (log m>3.61), who's structure can be characterized as a mass oscillator consisting of three spirals of two meanders, each of which contains $4 \ldots 5$ RASC units. Furthermore, in the collapsed form, it was assumed to stabilize the ends of polymer chains and thus ensure that the secondary structure of the protein has the lowest potential energy.

After $774 \mathrm{~h}$ the spectrum of small and middle domains was alike those of native collagens (Figures $\mathbf{3}$ and 4 ) although, with remarkable differences in the area of super micelle structures. With regard to small and middle domains, the similarity concerned only the expanded form and not the mass.

In Figure 8, the curves were given how the forms of NC1 hexamers and small spirals in gelatin changed at drying. As visible at the beginning of drying, both curves run synchronously, but after 270 $h$ the curve courses were changed to the opposite. The expanding of hexamers $(+f)$ and the collapsing of small spirals $(-f)$ at the drying end evidenced that small spirals do not belong to the structural elements of hexamers and the hexamers themselves are not part of the polymer chains in gelatin.

While the behavior of gelatin at drying has been investigated already in the following was of interest, how it behaves in the presence of water, especially in concentrated hydrogels where restructuring processes are of practical importance. In Figure 9, the emergence of collapsed mass structures in a concentrated gelatin hydrogel ( $20 \mathrm{wt}$. \%, $277 \mathrm{~K}$ ) was given.

As visible after $68 \mathrm{~h}$ the part of collapsed structures in the

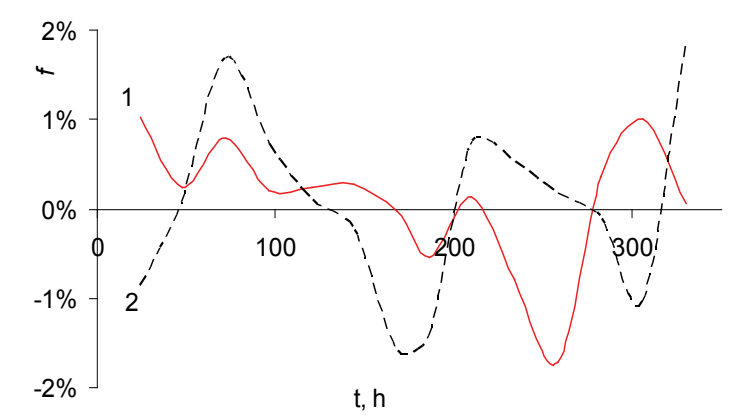

Figure 8 Changing domain forms in gelatin (Figure 6) at film drying in air at $343 \pm 20 \mathrm{~K} .1$ - NC1 hexamer $(170 \mathrm{kDa})$, 2- spiral (455 Da, Figure 4).

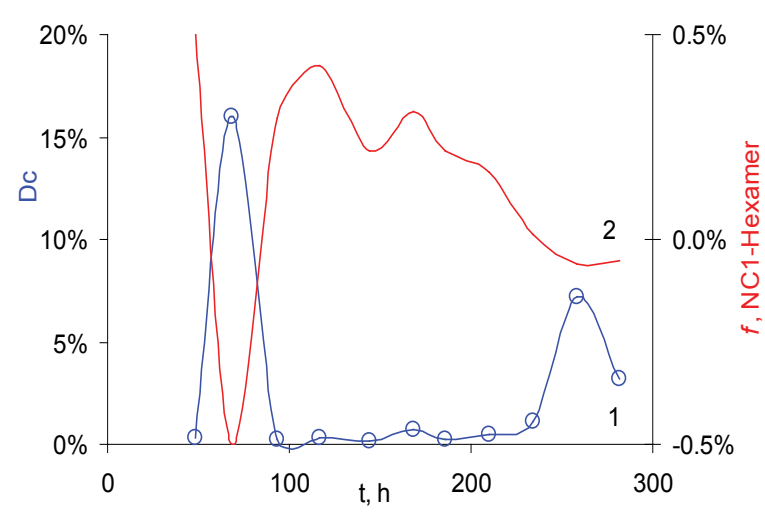

Figure 9 Emergency of collapsed mass concentrations in a concentrated gelatin hydrogel at 277 K. 1- part of polymer domains and water clusters in the collapsed form in the whole ensemble, 2- $f$ value for NC1 hexamers.

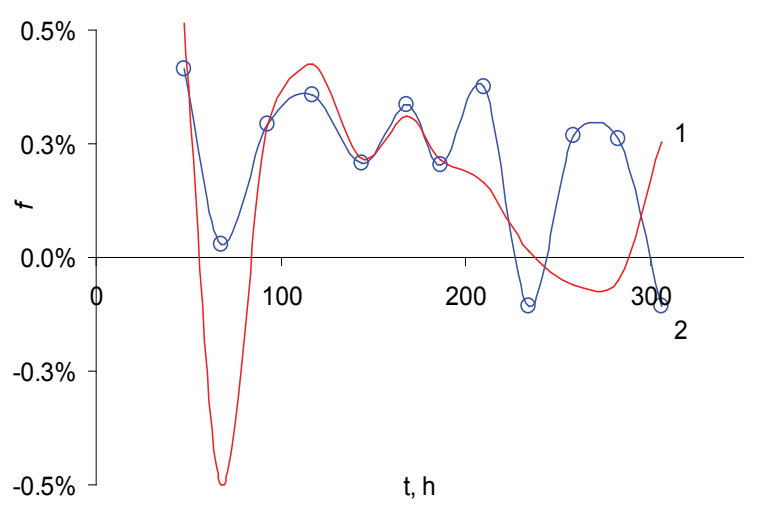

Figure 10

Change dynamics of NC1 hexamer (1) and NC1 dimer $(2,85 \mathrm{kDa})$ shapes in a $20 \%$ gelatin hydrogel at $277 \mathrm{~K}$.

hydrogel was the highest, in the NC1 hexamers the collapsed forms dominated too. At the beginning, the collapsed forms were temporarily in the most than the hexamers were again transformed into the expanded ones. Such dynamics can be understood when NC1 was influenced by restructuring secondary and higher structures of the polymer chains. Although NC1 was 


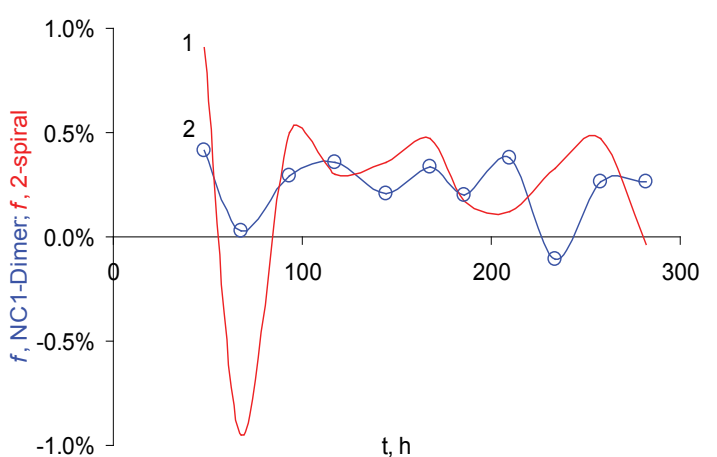

Figure 11

Change dynamics of domain shapes in the concentrated gelatin hydrogel at $277 \mathrm{~K}$. 1-spiral of 2 meanders, 2- NC1 dimer.

not part of the domain structure of the gelatin chains the hexamer belonged to the whole domain ensemble in the sample. This chains' restructuring forced to change the form of NC1. Further, inside the hexamer the events developed differently (Figures 10 and 11). Initially, the density (form) of domains changed synchronously, but in the later stages of the hydrogel ripening, relaxation processes in the higher chain structures disturb these curve courses. Upon reaching $300 \mathrm{~h}$ the hexamer was mainly in the expanded form, its dimer however in the collapsed. This phenomenon can be understood only under the influence of the polymorphic structures of water, which clusters also undergo restructuring at approaching a thermodynamic state with a new low potential energy [1].

Comparing the dynamics of the shape change of the NC1 dimers and small spirals it was striking that in the dimers were dominating expanded structures but in spirals collapsed (Figure 5), however, only at the beginning restructuring of supramolecular structures of the polymer chains, later they changed to the expanded states (Figure 11).

Unlike the NC1 hexamers and small spirals, the NC1 dimers reacted only weakly on restructuring in the supramolecular structure of protein chains, thus evidencing their high stability against conformational changes in small spirals. Hence these spirals were concluded not to be structural elements of the dimer, and lastly of the NC1 hexamer.

The signal at $\log m=3.5$ being one of the strongest signals could belong to both the water cluster of 179 molecules and the hypothetical domain (M) of 20 RASC (Figure 12). In hydrogels, it was found to change irregularly its form (density) that may be an indicator for specific rearrangement processes of secondary and higher structures in protein chains. The changes were observed in the whole range of studied mass ensembles of clusters and domains in hydrogel (Figure 12), but they did not concern the mass concentrations, which were allowed by the gravitational noises in our galaxy. As visible the ensemble of clusters and domains in hydrogel was represented by expanded structures mainly nevertheless, at aging, conversions from the collapsed form to the expanded one and conversely ran more than once.

So already at $68 \mathrm{~h}$ sub ensembles (indicated by arrows) that

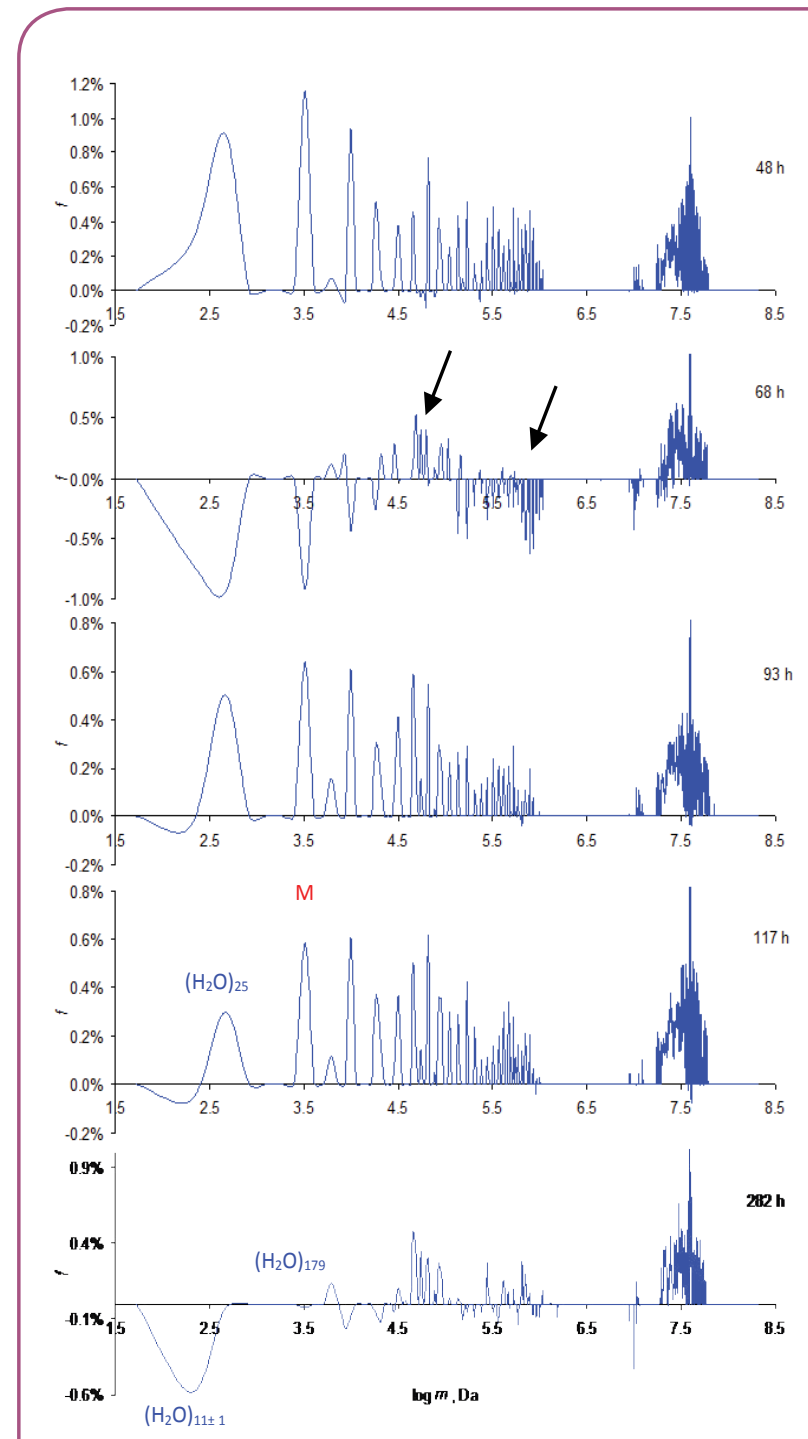

Figure 12

Change dynamics of cluster and domain parts in an ensemble of gelatin hydrogel at $277 \mathrm{~K} .48 \mathrm{~h}-\mathrm{M}_{\mathrm{GMS}}$ $28,619 \mathrm{kDa}, \mathrm{N}=458, \mathrm{Dc}=0.4 \% ; 68 \mathrm{~h}-\mathrm{M}_{\mathrm{GMS}} 28,291$ $\mathrm{kDa}, \mathrm{N}=439, \mathrm{D}_{\mathrm{c}}=16 \%$; $93 \mathrm{~h}-\mathrm{M}_{\mathrm{GMS}} 33,539 \mathrm{kDa}, \mathrm{N}=557$, $D_{c}=0.2 \% ; 117 \mathrm{~h}-\mathrm{M}_{\text {GMs }} 31,007 \mathrm{kDa}, \mathrm{N}=457, \mathrm{D}_{\mathrm{c}}=0.3 \%$; $282 \mathrm{~h}-\mathrm{M}_{\mathrm{GMS}} 31,496 \mathrm{kDa}, \mathrm{N}=423, \mathrm{D}_{\mathrm{c}}=3 \%$. The estimated mass of the Chaplin water cluster of 184 molecules [22] in the ensemble was here represented by the experimentally found water cluster of 179 molecules.

reflect the formation of large domain structures in hydrogel were detected, but they were still unstable and needed to be reconstructed under the influence of other surrounding domains. Thus, the ensemble of clusters and domains in hydrogel aged and was in search of a minimum potential energy of its state. Here the influence of a second element in hydrogel like a water cluster ensemble on the supramolecular structures of protein chains cannot be ignored hence, it makes sense to look at how the water morphology [1] (Figure 13) influenced the ensemble of protein domains in gelatin hydrogel (Figure 14).

Figure 13 showed the GMS spectra of water that was exposed to sounds of different frequencies. As visible the cluster structures differed from each other. These structures were found to be stable 

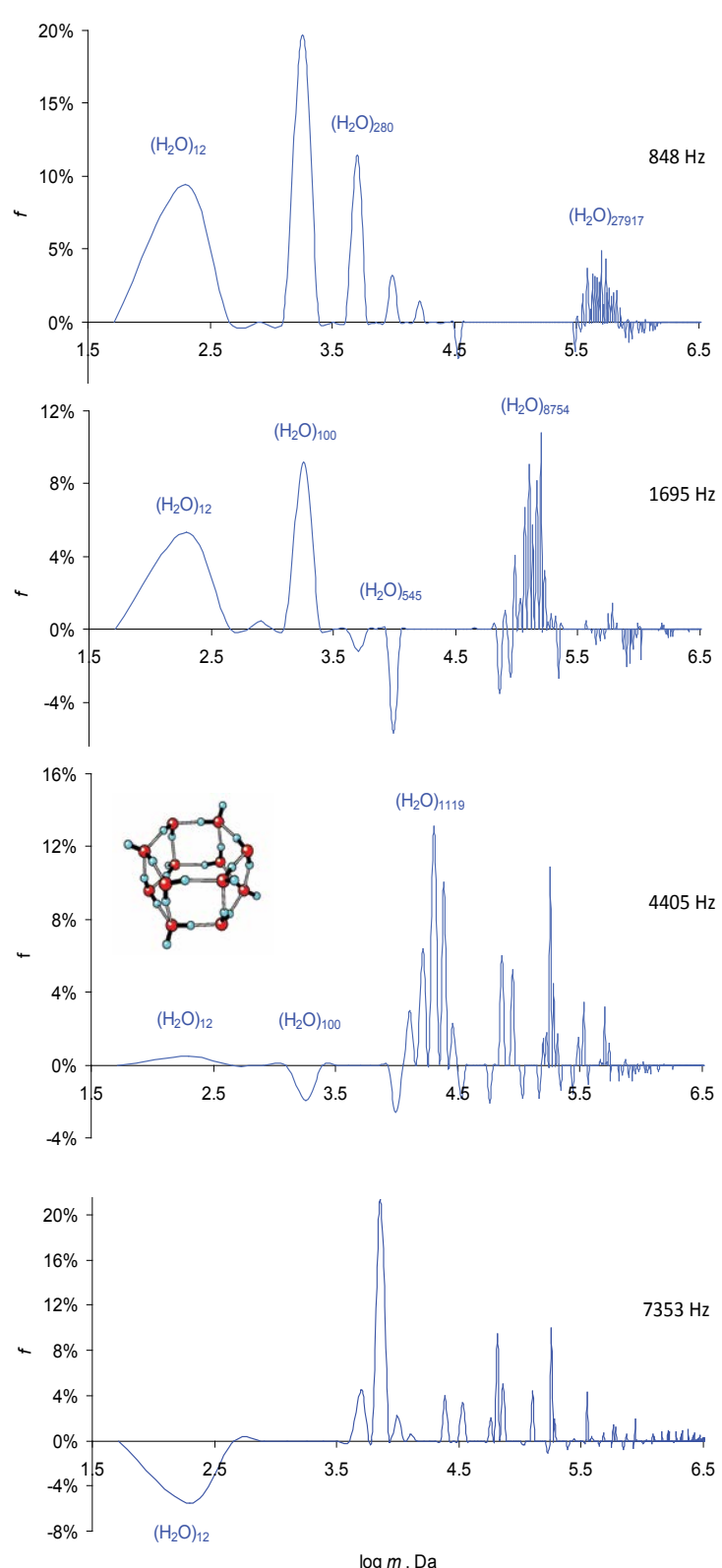

Figure 13 GMS spectra of polymorphous structures of water applied for this study.

The modeled base water cluster was kindly provided by A. Lenz [27]. See too: http://lenta.ru/ photo/2016/02/26/soundwaves/\#11

under sound exposure, only and when disconnecting the sound generator the morphology immediately changed leading to the same LRO. At low sound frequencies, the signal of the base water cluster $\left(\mathrm{H}_{2} \mathrm{O}\right)_{12}[26,27]$ got a positive $f$-value, which indicated its expanded structure [1]. At the frequency of the Chaplin cluster of 100 molecules at $7353 \mathrm{~Hz}$ (Table 1) the signal of the cluster even disappeared while the base water cluster became collapsed $-f[1]$.

Figure 14 showed a series of gelatin spectra at swelling, stimulation of specific LRO structures of water clusters in the swollen sample (at $7353 \mathrm{~Hz}$ ), and after soft drying. Here, at $7353 \mathrm{~Hz}$ a balance

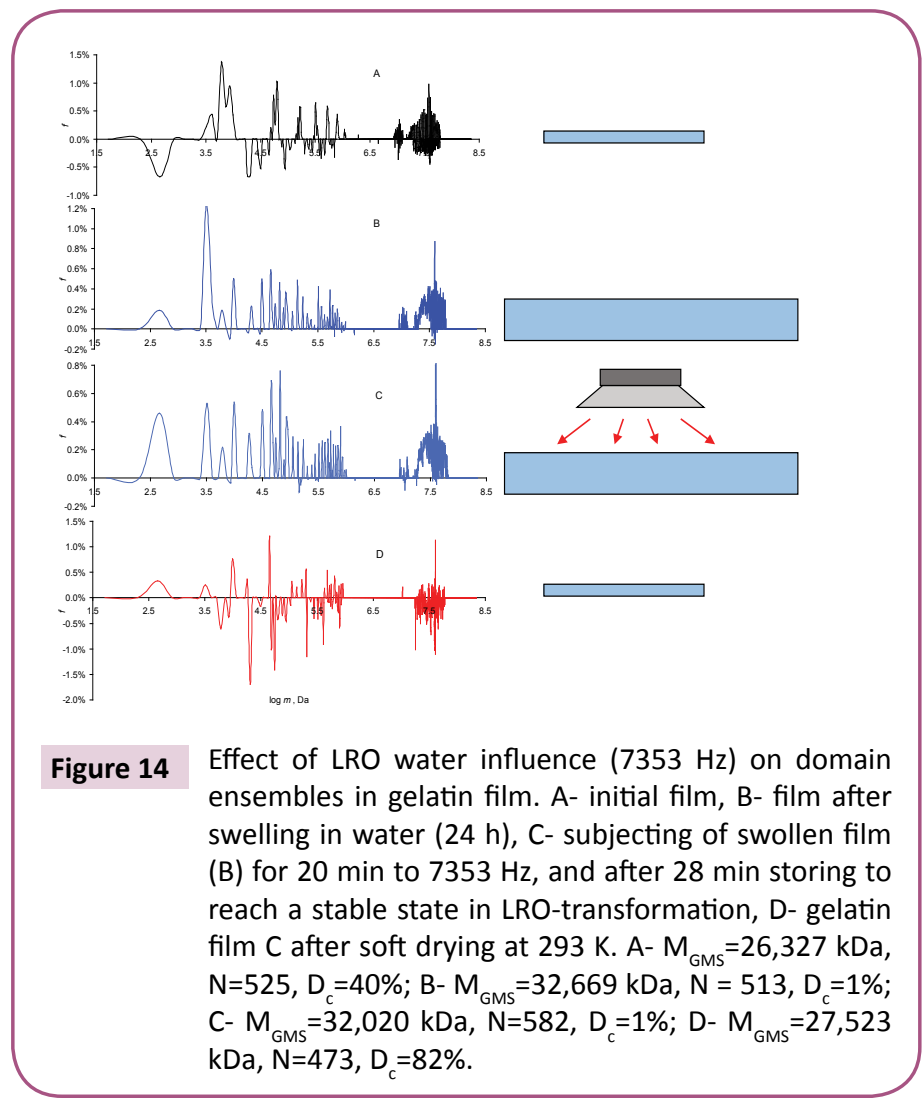

between collapsed and expanded structures of $\left(\mathrm{H}_{2} \mathrm{O}\right)_{100}$ was achieved no signal in the spectrum, consequently. Generally, the spectra highly differed from each other. Swelling and the impact of the amorphous water structure with dominating water clusters $\left(\mathrm{H}_{2} \mathrm{O}\right)_{100}$ [1] did not return the ensemble of protein domains in the initial state of collagen LRO, revealed especially for the sub ensembles of large domains ( $\log m>6.5)$. A tendency for a harmonization between collapsed and expanded supramolecular structures of polymer chains D, (Figure 14) was marginally noticeable. In the small mass concentration area, the signals from small spirals became expanded. As LRO of water with dominating $\left(\mathrm{H}_{2} \mathrm{O}\right)_{100}$ affected the supramolecular structure of the protein it was interesting to understand the behavior of other LROs.

The influence of other water LRO on domain ensembles in the polymer was shown in Figure 15. As follows LRO of water strongly influenced the domain structure in the hydrogel that can be used to change the polymer domain structure and later higher conformational states of the protein chains. The splash of the average molecular mass of all domains in the solid gelatin after stimulating by specific water LRO in the hydrogel $(4405 \mathrm{~Hz})$ could give an indication on an intensive development of seed crystals in the whole ensemble of investigated masses. Thus it seems to be possible using water LRO to approximate the gelatin LRO to that one of collagen. It should be noted however, that the effect depended strongly on the production process of gelatin.

Previously we found that mechanical deformations of liquids, polysaccharides and synthetic polymers influenced individual clusters, domains and domain ensembles in their analogues that 


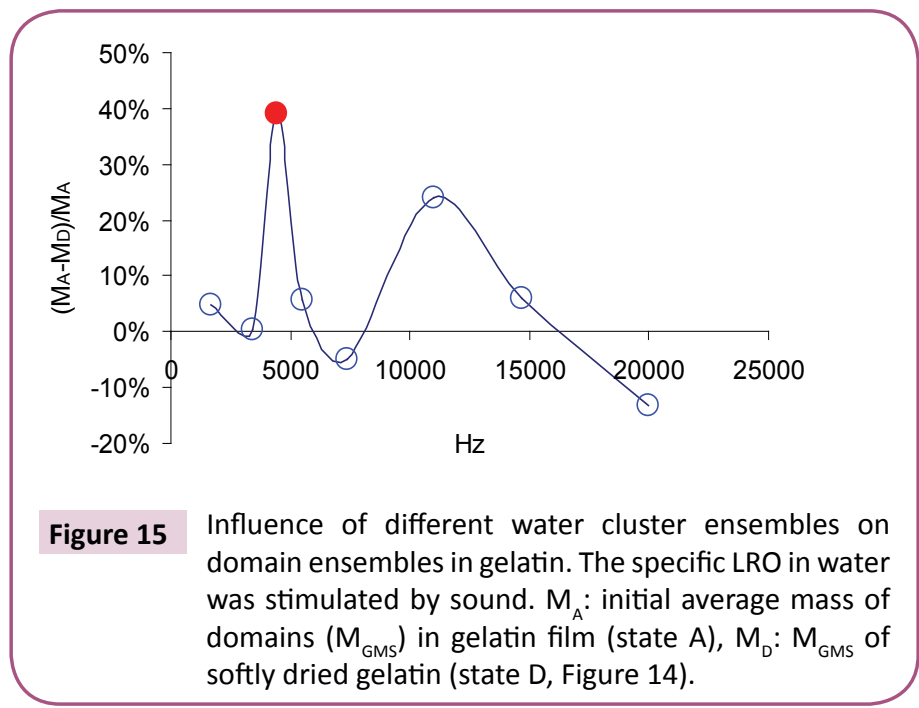

were at some distance from them [1]. It was of interest to test this phenomenon for proteins. Figure $\mathbf{1 6}$ showed the reaction of domain ensembles in gelatin located $30 \mathrm{~cm}$ from the sample analogue that was subjected to bending deformation (Figure 2).

As visible from Figure 16, the domain ensembles in protein responded to events in their analogues located at a distance of $30 \mathrm{~cm}$. Forcibly changing the potentially energy state of a domain ensemble in a protein film by mechanical deformations (5 s) caused after some seconds a LRO restructuring in the other domain ensembles. Thus, domains and domain ensembles of the same protein formed uniform resonance systems that reflect the common structure of the neutrino fields around mass assembles of the same substance. Applying this discovery it should be possible to influence distantly domains and hence the conformations of protein chains in biomatrices e.g. in rapidly mutating systems (viruses), in systems with abnormal tissue development (sprains, bruises, contraction and expansion of blood vessels). It creates a platform for future medicine without pills. Note that this phenomenon has been used in nature for a long time for example, subconscious scratching - a method to remove effects of tissue spasms. Physiotherapy (massage, heat) also belongs to harmonization of domain ensembles in biomatrices (Figure 17).

This treatment method as a way of minimizing the potential energy inside a single oscillator (mass ensembles of similar material systems) should not be confused with the hypothetical teleportation (https://en.wikipedia.org/wiki/Teleportation)

\section{Conclusion}

Long-range order in collagen and gelatin was represented by ensembles of atomic nuclei mass concentrations - domains and super domain formations. Domain ensembles were not constant, but dynamic systems that reflected the state of supramolecular structures of protein chains. Domain ensembles in collagens and gelatins actively influenced the cluster ensembles in water and vice versa. Between the two ensembles (water \& protein) existed a thermodynamic equilibrium affecting the supramolecular

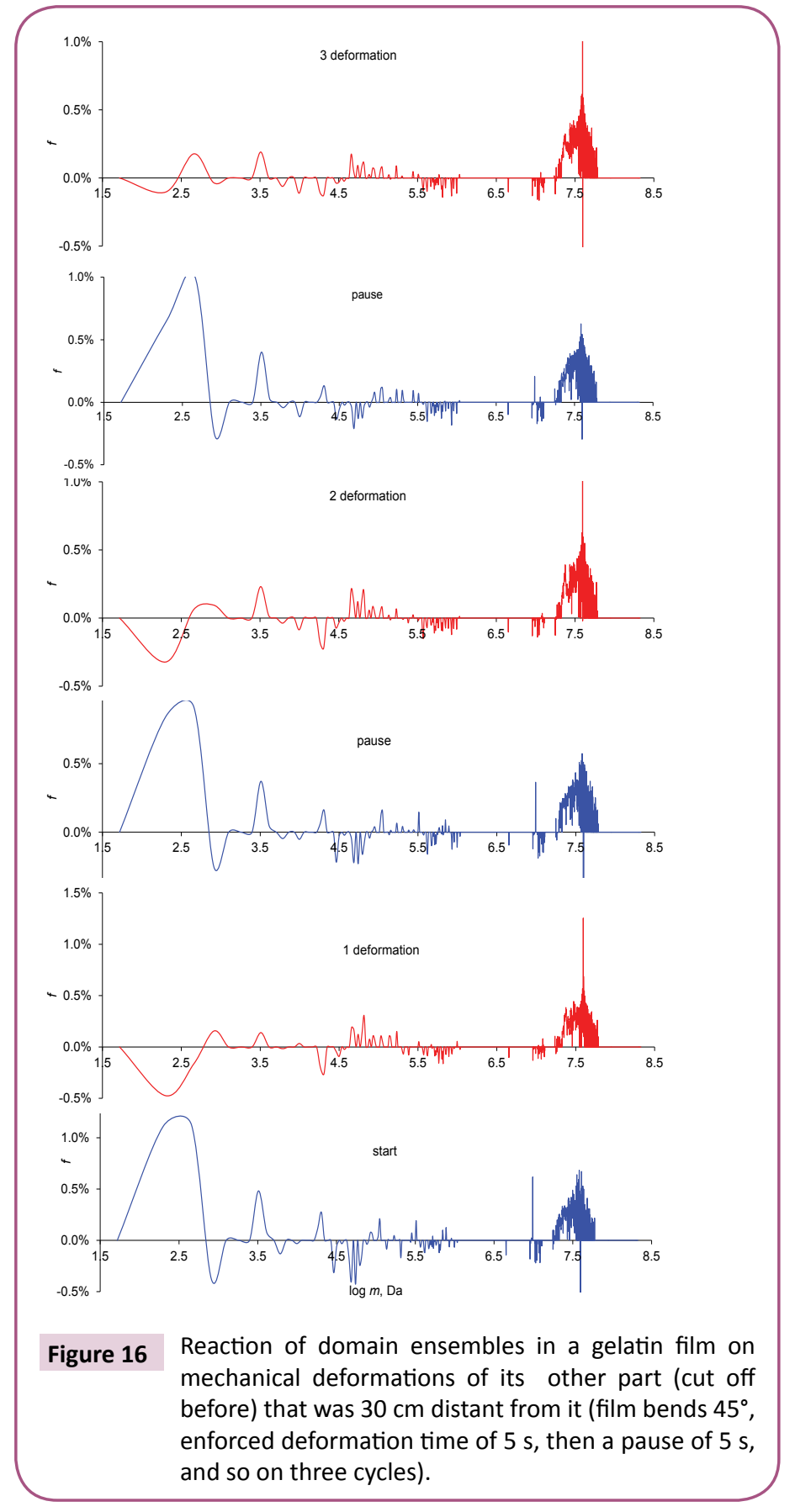

structure of the polymer chains. The cluster structure of water and its long-range order was found to be part of the long-range order of domains in gelatins and collagens.

To get the domain structure of native collagens from industrial gelatins was impossible. Domains and domain ensembles of proteins distantly interacted with each other and were in a common resonance gravitational field of neutrinos in our sun system. This effect should be a platform for a new biochemistry medicine in the $21^{\text {st }}$ century.

\section{Sponsorship}

For a further project developing the authors are looking for sponsors. Please contact us. 


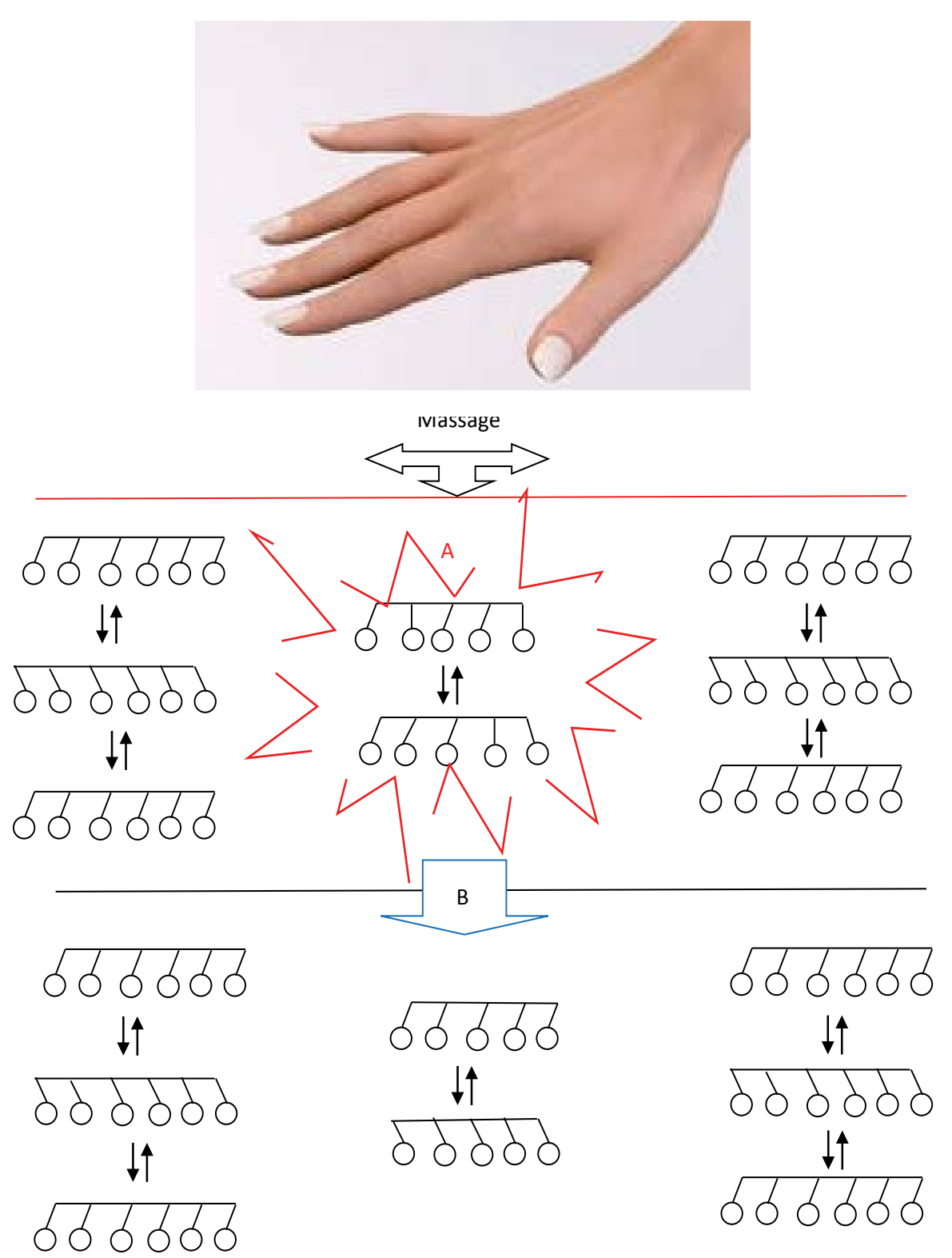

Figure 17 Modeled enforced harmonization of an inharmonic oscillator ensemble (A- fragment of a pathological biomatrix) inside an ensemble of harmonic oscillators (B-healthy biomatrix) under influence of many pendula (domain ensembles) from surroundings (blue pendulum balls) at massages. 


\section{References}

1 Zubow K, Zubow AV, Zubow VA (2014) The Way to the ETIs. Applied gravitational mass spectroscopy. Nova Science Publishers, United States.

2 Sato ET, Rocha AR, de Carvalho LF, Almeida JD, Martinho H (2015) Molecular model for hydrated biological tissues. Phys Rev E Stat Nonlin Soft Matter Phys 91: 1-6.

3 Khiari Z, Ndagijimana M, Betti M (2014) Low molecular weight bioactive peptides derived from the enzymatic hydrolysis of collagen after isoelectric solubilization/precipitation process of turkey byproducts. Poult Sci 93: 2347-2362.

4 Kazanci M (2014) Solvent and temperature effects on folding of electrospun collagen nanofibers. Materials Letters 130: 223-226.

5 Brand I, Habecker F, Ahlers M, Klüner T (2015) Structure of collagen adsorbed on a model implant surface resolved by polarization modulation infrared reflection-absorption spectroscopy. Spectrochim Acta A Mol Biomol Spectrosc 138: 216-224.

6 Lipscaomb D, Echchgadda I, Ibey BL, Beier H, Thomas RJ, et al. (2012) Terahertz spectroscopy of dry, hydrated, and thermally denatured biological macromolecules. Proceedings of SPIE 822: 1-12.

7 Ooms KJ, Vega AJ, Polenova T, Cannella M, Marcolongo M (2015) Double and zero quantum filtered ${ }^{2} \mathrm{H} N M R$ analysis of $\mathrm{D}_{2} \mathrm{O}$ in intervertebral disc tissue. J Magn Reson 258: 6-11.

8 Eklouh-Molinier C, Happillon T, Bouland N, Fichel C, Diébold MD, et al. (2015) Investigating the relationship between changes in collagen fiber orientation during skin aging and collagen/water interactions by polarized-FTIR microimaging. Analyst 140: 6260-6268.

9 Rodin VV, Nikerov VA (2014) NMR-Relaxation and PFG NMR studies of water dynamics in oriented collagen fibres with different degree of cross-linking. Current Tissue Engineering 3: 47-61.

10 Guo J, Zhang W, Lia Xuepin, Shi B (2012) Near infrared and twodimensional correlation infrared spectroscopic study on the heat denaturation of collagen in aqueous solution. Journal of the American Leather Chemists Association 107: 205-212

11 Belitz HD, Grosch W, Schieberle P (2008) Lehrbuch der Lebensmittelchemie. Springer-Verlag, Germany.

12 Vanderschee CR, Ooms KJ (2014) Investigating Water interactions with collagen using $2 \mathrm{~h}$ multiple quantum filtered $\mathrm{nmr}$ spectroscopy to provide insights into the source of double quantum filtered signal in tissue. J Phys Chem B 118: 3491-3497.

13 Gevorkian SG, Allahverdyan AE, Gevorgyan, DS, Simonian Aleksandr, Hu CK (2013) Stabilization and anomalous hydration of collagen fibril under heating. PLoS One 8: e78526.
14 Zubow K, Zubow AV, Zubow VA (2014) Molecular cluster ensembles in biomatrices and the influence of etis. New way to the spread of life in space. Advances in Chemistry Research 22: 1-49.

15 Guo J, Zhang W, Liao X, Shi B (2012) Near infrared and two-dimensional correlation infrared spectroscopic study on the heat denaturation of collagen in aqueous solution. Journal of the American Leather Chemists Association 107: 205-212

16 Fathima NN (2011) Hydration and shrinkage phenomena in native and crosslinked collagen matrices. Proceedings of the Indian National Science Academy 77: 283-294

17 De Simone A, Vitagliano L, Berisio R (2008) Role of hydration in collagen triple helix stabilization. Biochemical and Biophysical Research Communications 372: 121-125.

18 Zubow KV, Zubow AV, Zubow VA (2010) Ensemble of Clusters - New Form of Molecular Matter, Risks and chances. Zubow equations. In Advances in Chemistry Research 5: 107-145.

19 Chaplin M (2016) Water Structure and Science, SBU, London.

20 Than ME, Henrich S, Huber R, Ries A (2002) The 1.9- a crystal structure of the noncollagenous (NC1) domain of human placenta collagen IV shows stabilization via a novel type of covalent Met-Lys cross-link. Proc Natl Acad Sci USA 99: 6607-6612.

21 Ninichuk V (2008) The role of chemokine receptor CCR1-dependent macrophage recruitment for the progression of chronic kidney disease in murine Alport syndrome or type 2 diabetes. Dissertation. Medizinischen Fakultät der Ludwig-Maximilians-Universität zu München. Muenchen.

22 Ries A, Engel J, Lustig A, Kühn K (1995) The Function of the NC1 Domains in Type IV Collagen. J of Biological Chem 270: 23790-23794.

23 Fidler AL, Vanacore RM, Chetyrkin SV, Pedchenko VK, Bhave G, et al. (2014) A unique covalent bond in basement membrane is a primordial innovation for tissue evolution. PNAS 111: 331-336.

24 Faller M, Matsunaga M, Yin S, Loo JA, Guo F (2007) Heme is involved in microRNA processing. Nature Structural \& Molecular Biology 14: pp.23-29.

25 https://www.google.de/search?q=Tertiary+structure+of+collagen+p icture \&biw $=1366 \&$ bih $=633 \& \mathrm{tbm}=$ isch $\&$ tbo $=u \&$ source $=u n i v \& s a=X \&$ ved=0ahUKEwjwibz2mu3LAhWD1ywKHdgkD-8QsAQIIA

26 Bogdanov EV, Manturova GM (2000) Equicuster water model. Biomedical Electronics 7: 19-28.

27 Lenz A, Ojamäe L (2006) On the stability of dense versus cage-shaped water clusters: Quantum-chemical investigations of zero-point energies, free energies, basis-set effects and IR spectra of $\left(\mathrm{H}_{2} \mathrm{O}\right)_{12}$ and $\left(\mathrm{H}_{2} \mathrm{O}\right)_{20}$. Chem Phys Letters 418: 361-367. 